\title{
Développements récents en matières de raffinage et de modifications : élimination des contaminants dans les huiles alimentaires et réduction du taux d'acides gras trans
}

\author{
Jan DE KOCK \\ Wim DE GREYT \\ Véronique GIBON \\ Marc KELLENS

\section{Desmet Ballestra,} \\ de smet technologies \& services, \\ Da Vincilaan, 2, Bus G1, \\ B-1935 Zaventem, Belgique \\ fax : +3227161109 \\ <dgw@desmetgroup.com>
}

\begin{abstract}
As a result of the general concern for food safety, the removal of minor contaminants (PAH, pesticides, dioxins, $P C B^{\prime}$ '...) from oils and fats has the be examined in detail.

Also, in recent years a lot of attention has been given to the lowering of the trans fatty acids content in oils and fats for food products; the reason for this move is the potential negative health impact of trans fatty acids.

In general, contaminants can be removed either during neutralization, by adsorption on activated carbon or during the deodorization process. To reach an efficient removal, the process conditions applied need to be optimised to achieve almost complete detoxification but with minimal costs.

Traditionally, partially hydrogenated fats were used in nearly all food products because of their interesting melting properties. Nowadays, these products are replaced by trans free alternatives in most food products (margarines, bakery products...).

This has created renewed interest for other modification processes: multiple dry fractionation and chemical and enzymatic interesterification. The challenge is to produce trans free fats with the same functionality as the trans rich products, but at comparable cost.
\end{abstract}

Key words: edible oils, contaminants, trans fatty acids, refining, modification processes

par des acides gras insaturés cis plutôt que par des acides gras saturés.

L'impact nutritionnel des huiles et graisses toujours fait l'objet de beaucoup d'attention. Les matières grasses constituent en effet l'une des sources énergétiques principale en alimentation humaine. À côté de l'aspect énergétique, les graisses sont aussi vectrices de vitamines, d'acides gras essentiels et autres constituants mineurs, tous bénéfiques pour notre santé.

Ces dernières années, la pression s'est particulièrement renforcée en matière de sécurité alimentaire et les matières grasses n'échappent pas à cette contrainte.

Un sujet particulièrement sensible concerne la présence d'agents contaminants: une substance chimique ne devient contaminante que lorsqu'elle comporte un risque pour la santé humaine. Les contaminants chimiques proviennent soit de la pollution industrielle, soit de pratiques mal maîtrisées de l'agriculture ou de I'industrie alimentaire.

Un autre sujet de discussion concerne la réduction des acides gras trans (AGT) dans notre alimentation : un certain nombre de mesures législatives ont déjà été ordonnées visant à limiter la consommation de ces acides. Les AGT augmentent en effet le risque de maladies cardiaques et la nécessité de n'en consommer que de faibles quantités, quelle que soit leur origine, est bien établie. La tendance vers une réduction ou une suppression de ces acides gras dans de nombreux produits alimentaires se poursuit, avec un remplacement préférentiel

\section{Agents contaminants}

On retrouve dans la plupart des huiles brutes des composants mineurs à effet contaminant que l'on appelle classiquement polluants organiques persistants ou POP. Ceux-ci regroupent principalement les pesticides chlorés (DDT, toxaphène, aldrine, etc.), les hydrocarbures polycycliques aromatiques (HPA), les dioxines, furanes et biphényles polychlorés ( $P C B$ ) ainsi que les retardeurs de flammes polybromés (diphényléthers polybromés).

Le caractère persistant des POP lié à une stabilité chimique importante entraîne leur accumulation dans la chaîne alimentaire ; la lyposolubilité élevée de ces molécules favorise en outre leur concentration dans les huiles et graisses alimentaires. II est clair que les POP présentent une toxicité non négligeable et qu'ils sont caractérisés par de faibles doses journalières tolérables; la limite maximale de résidus autorisée dans les matières grasses est en conséquence très faible.

\section{Raffinage}

L'objectif principal du raffinage d'une huile est de réduire son contenu en éléments mineurs non triglycérididiques (phospholipides, métaux, acides gras libres, savons, pig- ments, produits d'oxydation...) qui ont un effet néfaste sur sa qualité en termes de stabilité oxydative. II convient par ailleurs de ne pas endommager la fraction triglycéridique (polymérisation, transisomérisation, etc.) et de conserver un maximum de constituants reconnus comme bénéfiques (tocophérols, tocotriénols, stérols, etc.).

Le processus de raffinage comprend une série d'étapes distinctes qui peuvent s'opérer de deux manières différentes: le raffinage chimique et le raffinage physique. En raffinage chimique, les acides gras libres et la plupart des phospholipides et autres impuretés sont enlevés dans l'étape de neutralisation alcaline ; une étape séparée de dégommage n'est donc pas nécessaire. En raffinage physique, les acides gras libres sont éliminés par une distillation conjointe à la désodorisation ; une étape préalable de dégommage est en conséquence indispensable.

Il est clair que, quelle que soit la voie choisie (chimique ou physique), le procédé de raffinage doit être adapté pour permettre en outre une élimination optimale des composants mineurs à effet contaminant.

Certains constituants peuvent être éliminés dans l'étape de neutralisation. Mais ce sont surtout les étapes de décoloration (adsorption sélective) et de désodorisation qui permettent de réduire au maximum le niveau de contaminants. 


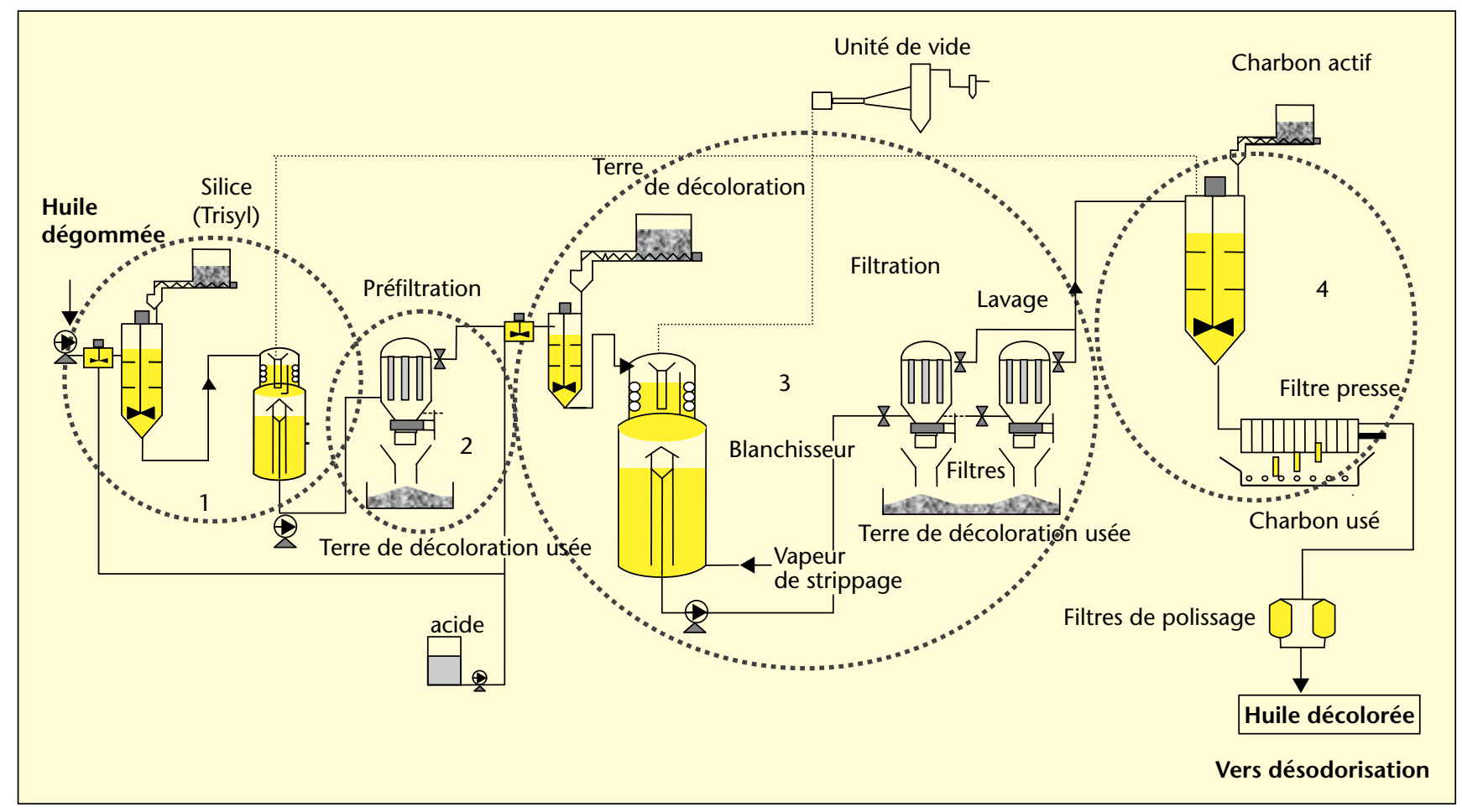

Figure 1. Procédé Combiclean : procédé modulaire à multi-étapes combinant le prétraitement, la décoloration et la détoxification.

La contamination aux hydrocarbures polycycliques aromatiques (HPA) a souvent lieu en cours de séchage direct à la fumée des fruits ou des graines (coprah, palmiste, colza, etc.). II convient de distinguer les HPA lourds des HPA légers, principaux constituants de cette fraction. Les HPA lourds ne peuvent être extraits que par adsorption sur charbon actif, dont certains sont spécialement développés à cet effet. Le niveau en HPA lourds atteint aux termes de cette étape est inférieur à $5 \mathrm{ppb}$. Les HPA légers sont quant à eux suffisamment volatils que pour être entrainés lors de la désodorisation. Pour du coprah par exemple, les conditions conventionnelles $\left(210-235^{\circ} \mathrm{C}, 2-4\right.$ mbar) sont tout à fait adaptées pour une réduction du taux de HPA total en dessous de 20-25 ppb, ce qui est la teneur maximale généralement admise dans les spécifications d'une huile raffinée. Des niveaux supérieurs à 25 ppb peuvent être parfois détectés mais ils correspondent à des huiles brutes particulièrement contaminées.

Une incinération incorrecte des déchets est la source majeure de pollution à la dioxine; celle-ci est assez facile à éliminer des huiles (> $90 \%$ ) par adsorption sur charbon actif. Les $P C B$, utilisés dans les transformateurs et les condensateurs comme fluide hydraulique, comme liquide de refroidissement et pour diverses autres applications (laques, matières synthétiques...) sont caractérisés par une grande stabilité, une liposolubilité importante et une mauvaise biodégradabilité ; ceci a pour conséquence un maintien permanent dans I'environnement et une accumulation importante dans la chaîne alimentaire. Les PCB sont plus difficiles à adsorber sur charbons actifs. Vu leur volatilité relativement élevée, ils sont éliminés de façon plus efficace lors de l'étape de désodorisation réalisée dans des conditions optimalisées.

Les pesticides chlorés sont éliminés intégralement en désodorisation, pour autant que la température soit au minimum de $230^{\circ} \mathrm{C}$ et la pression inférieure à 4 mbar. Vu leur haute volatilité, la plupart des pesticides sont distillés ; cependant, certains pesticides (captan, dichlovos, etc.) sont décomposés à la chaleur en produits plus volatils eux-mêmes distillés. Dans des conditions normales de travail, il est aisé d'atteindre des seuils résiduels de l'ordre de 10 à 50 ppb en pesticides chlorés.

\section{Innovations technologiques}

Les innovations destinées à répondre aux nouvelles attentes prennent en conséquence deux orientations: la recherche de procédés $d^{\prime}$ adsorption plus efficaces et l'élaboration de technologies de désodorisation mieux adaptées.

\section{Procédés d'adsorption plus efficaces}

Le procédé classique de décoloration en une seule étape a été modifié en un procédé modu- laire à multi-étapes combinant le prétraitement, la décoloration et la détoxification (procédé Combiclean) (figure 1).

Ce procédé comprend conventionnellement quatre étapes consécutives :

1) le prétraitement à la silice ;

2) la préfiltration sur filtre chargé de terres décolorantes usées ;

3) la décoloration elle-même ;

4) le traitement au charbon actif.

La décoloration en tant que telle reste l'étape clef du procédé Combiclean.

Le prétraitement à la silice et la préfiltration sur terres décolorantes usées constituent une réelle amélioration du procédé tant au plan économique qu'au plan qualitatif. Ce prétraitement permet l'élimination de certains composants mineurs indésirables (tels que les phospholipides, les savons, etc.). Il permet aussi une réduction importante de la consommation de terres décolorantes (20-30\%).

Le traitement au charbon actif est indispensable pour éliminer les contaminants non volatils tels que les HPA lourds et les dioxines.

La séparation complète du traitement à la terre et au charbon actif génère deux types de sousproduits : une terre décolorante usée non toxique et un charbon actif usé, en principe toxique. Cette séparation peut être importante (mais n'est pas indispensable), que ce soit dans le cas d'une valorisation ultérieure ou d'une mise à la décharge. Par ailleurs, elle permet une activité optimale du charbon actif (dosage 


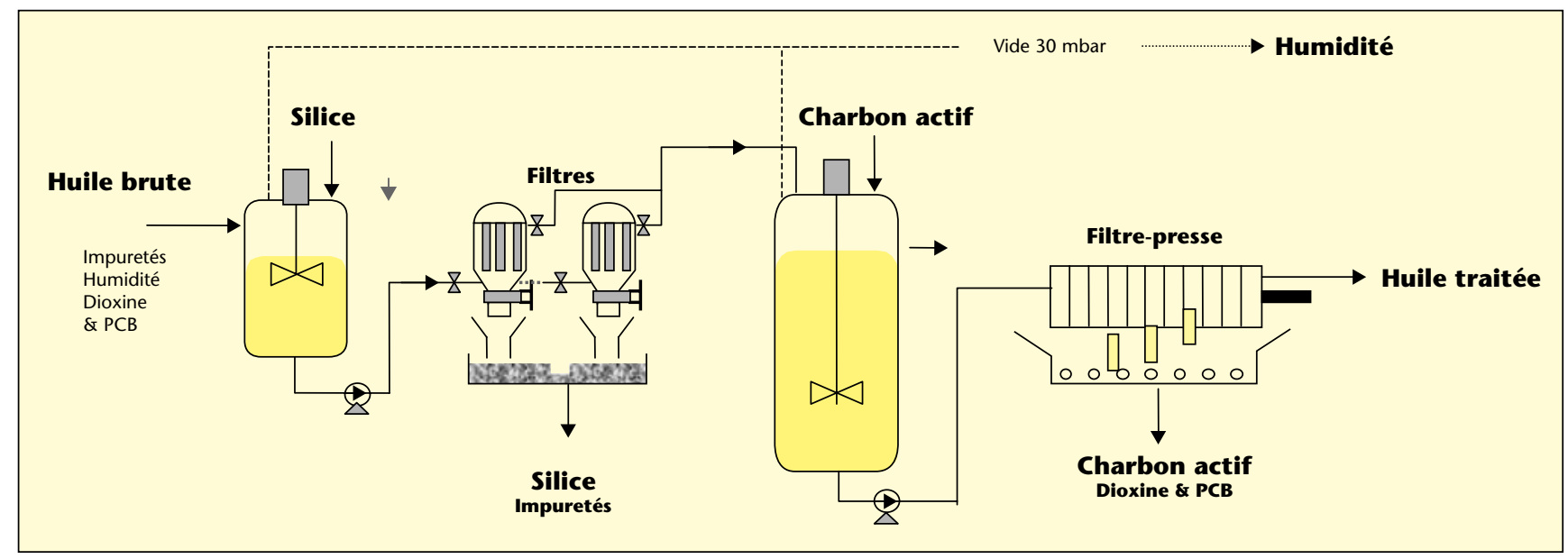

Figure 2. Unité de détoxification d'huile de poisson brute par traitement au charbon actif.

Tableau 1. Niveaux de $P C D D, P C D F$, non-ortho et mono-ortho $P C B$ (exprimés en ppt $T E Q^{*}$ ) dans I'huile brute de foie de morue et dans les échantillons traités au charbon actif [1].

\begin{tabular}{|lccccccc|}
\hline Type de charbon actif & $\begin{array}{c}\text { Huile } \\
\text { brute }\end{array}$ & A & A & B & B & C & C \\
\hline Quantité de charbon actif (\%) & - & $\mathbf{0 , 1}$ & $\mathbf{0 , 5}$ & $\mathbf{0 , 1}$ & $\mathbf{0 , 5}$ & $\mathbf{0 , 1}$ & $\mathbf{0 , 5}$ \\
PCDD + PCDF totaux (ppt TEQ) & 5,41 & 0,80 & 0,17 & 0,89 & 0,09 & 0,43 & 0,00 \\
Pourcentage extrait (\%) & - & 85 & 97 & 83 & 98 & 92 & 100 \\
PCB non-ortho (ppt TEQ) & 14,32 & 10,84 & 3,42 & 10,83 & 4,06 & 8,54 & 0,93 \\
PCB mono-ortho (ppt TEQ) & 3,74 & 3,62 & 3,14 & 3,33 & 2,99 & 3,50 & 2,69 \\
PCB totaux (ppt TEQ) & 18,06 & 14,46 & 6,56 & 14,16 & 7,05 & 12,04 & 3,62 \\
Pourcentage extrait (\%) & - & 20 & 64 & 22 & 61 & 33 & 80 \\
\hline
\end{tabular}

* TEQ : toxicité équivalente.

Tableau 2. Niveaux de PCDD, PCDF, PCB non-ortho et mono-ortho (exprimés en ppt TEQ*) dans une huile de poisson brute, dans des échantillons désodorisés en conditions douces et dans des échantillons traités au charbon actif puis désodorisés [2]

\begin{tabular}{|c|c|c|c|c|c|c|}
\hline & $\begin{array}{l}\text { Huile de } \\
\text { poisson } \\
\text { brute }\end{array}$ & $\begin{array}{c}\text { Huile de } \\
\text { poisson } \\
\text { désodorisée } \\
\text { à } 180^{\circ} \mathrm{C}\end{array}$ & $\begin{array}{l}\text { Huile de } \\
\text { poisson } \\
\text { désodorisée } \\
\text { à } 190^{\circ} \mathrm{C}\end{array}$ & $\begin{array}{c}\text { Huile de } \\
\text { poisson traitée } \\
\text { au charbon } \\
\text { actif }\end{array}$ & $\begin{array}{l}\text { Huile de } \\
\text { poisson } \\
\text { traitée et } \\
\text { désodorisée } \\
\text { à } 180^{\circ} \mathrm{C}\end{array}$ & $\begin{array}{l}\text { Huile de } \\
\text { poisson } \\
\text { traitée et } \\
\text { désodorisée } \\
\text { à } 190^{\circ} \mathrm{C}\end{array}$ \\
\hline $\begin{array}{l}\text { PCDD + PCDF } \\
\text { totaux } \\
\text { (ppt TEQ) }\end{array}$ & 15,0 & 8,8 & 6,5 & 1,1 & 0,3 & 0,2 \\
\hline $\begin{array}{l}\text { PCB non- } \\
\text { ortho } \\
\text { (ppt TEQ) }\end{array}$ & 8,4 & 4,5 & 3,1 & 5,0 & 2,6 & 1,7 \\
\hline $\begin{array}{l}\text { PCB mono- } \\
\text { ortho } \\
\text { (ppt TEQ) }\end{array}$ & 3,4 & 1,6 & 1,1 & 3,2 & 1,5 & 0,9 \\
\hline
\end{tabular}

* TEQ : toxicité équivalente.

minimal) ainsi qu'une flexibilité maximale du procédé (appliqué seulement si nécessaire).

\section{Cas particulier de l'huile de poisson}

L'huile de poisson est une source exceptionnelle d'acides gras polyinsaturés de type omega-3 (EPA et DHA) ainsi que de vitamines liposolubles; certains lots souffrent malheureusement d'une contamination élevée en dioxines, furanes et en PCB. Dans ce cas, le défi majeur est d'éliminer sélectivement les agents contaminants sans altérer les propriétés nutritionnelles de l'huile ni sa qualité en termes de stabilité oxydative.
Le traitement au charbon actif semble l'option la plus efficace (figure 2). Les résultats d'une étude menée sur une huile de foie de morue de la mer Baltique hautement contaminée en dioxines (PCDD), furanes (PCDF) et PCB [1] sont présentés au tableau 1. II apparait que la fraction PCDD/PCDF est quasi totalement éliminée par adsorption sur $0.5 \%$ de charbon actif et que 60 à $80 \%$ des $P C B$ peuvent être sélectivement extraits. L'efficacité est bien sûr liée à la nature du charbon actif utilisé ; par ailleurs, l'affinité de l'isomère mono-ortho PCB semble plus faible que celle des analogues nonortho, probablement en raison de sa structure non coplanaire. Une désodorisation ultérieure réalisée à basse température $\left(180-190{ }^{\circ} \mathrm{C}\right)$ [2] dans des conditions optimalisées permet de réduire encore le taux de contaminants et de le porter en dessous du seuil toléré, sans altération des caractéristiques de I'huile (tableau 2). La quantité résiduelle tolérée (limite maximale en résidu) pour les dioxines, telle que fixée par la Commission européenne à partir de 2006, est de 4,0 ppt TEQ (toxicité équivalente) pour I'huile de poisson brute (utilisée en alimentation animale) et de 1,5 ppt TEQ dans I'huile de poisson raffinée (utilisée en alimentation humaine).

\section{Des technologies de désodorisation mieux adaptées}

Comme nous venons de le voir, les besoins actuels en matière de raffinage sont de plus en plus liés à des objectifs de détoxification d'une part et de préservation optimale des éléments nutritionnels d'autre part.

Pour répondre à ces objectifs qui peuvent paraître ambitieux, de nouvelles unités de production de vide (condensation à sec) pouvant atteindre une pression absolue très basse ( 1 mbar) dans le désodoriseur, ont été mises au point. Une pression absolue basse permet une 


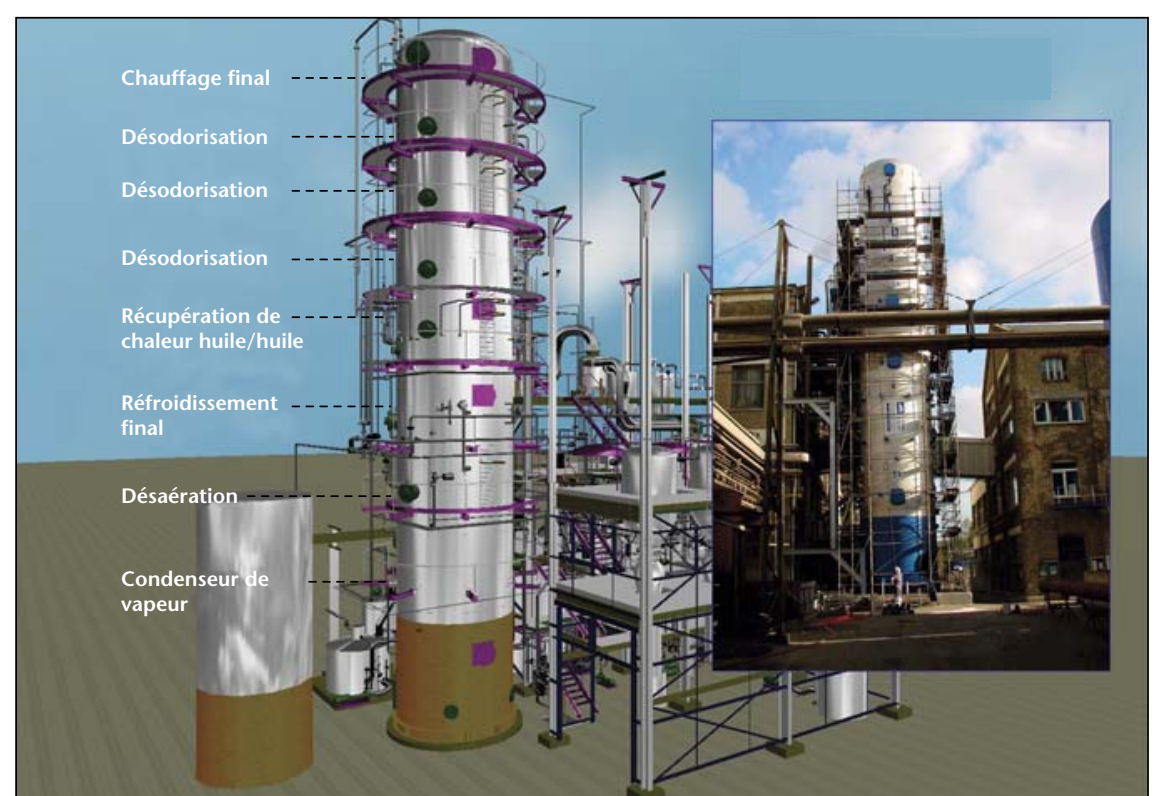

Figure 3. Technologie de désodorisation mieux adaptée aux besoins actuels : le Qualistock.

réduction de la température de désodorisation sans affecter l'efficacité de l'entraînement à la vapeur.

Plus précisément, le développement de désodoriseurs capables de travailler à des températures multiples a permis d'établir un compromis idéal entre le temps de séjour requis pour la désodorisation (réalisée à basse température) et celui nécessaire au blanchiment thermique et à la distillation (réalisés à température plus élevée). Le temps de séjour à haute température est réduit et fait de ces désodoriseurs un bon choix s'il convient d'éliminer les contaminants (HPA, PCB, etc.) ou de contrôler l'entrấnement de composants mineurs à haute valeur ajoutée (tocophérols, etc.) dans des huiles sensibles à la chaleur. Cette sensibilité est principalement liée à la formation d'isomères trans et à la polymérisation; la transisomérisation des acides gras polyinsaturés cis est considérablement réduite puisque le temps de séjour à haute température est particulièrement raccourci pour une efficacité optimale.

Des installations de désodorisation incluant toutes ces particularités sont actuellement disponibles en une tour unique pour installation en plein air (figure 3).

\section{Les isomères trans}

Les techniques actuelles en matière de désodorisation permettent donc de réduire au mieux le taux d'isomères trans produits dans les conditions de travail: le seuil actuellement acceptable est inférieur à $1 \%$ pour des huiles riches en acide $\alpha$-linolénique comme le soja ou le colza et à $0,5 \%$ pour les autres huiles (tournesol ou maïs).

\section{Techniques de modification}

\author{
Hydrogénation
}

La tendance actuelle en matière d'hydrogénation est une adaptation des conditions opérationnelles permettant de mettre sur le marché un produit fini appauvri en isomères trans.

Classiquement, l'huile de soja peut être hydrogénée à différents niveaux $d^{\prime}$ indices d'iode en fonction de son utilisation. Dans tous les cas, la quantité d'acides gras trans produite est importante (jusqu'à $50 \%$ pour certaines margarines et $65 \%$ pour les graisses d'enrobage) et ce, en relation directe avec le degré d'hydrogénation requis [3].

Les paramètres opérationnels (température et pression en hydrogène) peuvent être optimisés de sorte à réduire considérablement ce taux $d$ 'isomères trans tout en gardant l'indice $d^{\prime}$ 'iode relativement constant.

Dans le cas de l'huile de soja par exemple, une température basse et une pression élevée en hydrogène permettent de réduire notablement le taux d'isomères trans (figure 4 et tableau 3) [4].

L'emploi de catalyseurs plus sélectifs et l'amélioration de l'efficacité de l'agitation sont également des facteurs à prendre en considération pour une réduction optimale des acides gras trans. Ces améliorations demeurent cependant coûteuses ce qui limite encore leur application. Dans l'élaboration de nouveaux produits à bas taux d'isomères trans ( $<5-10 \%)$, il est à présent envisageable de formuler des mélanges d'huiles partiellement hydrogénées dans des conditions optimalisées et de graisses non hydrogénées d'origine animale ou végétale (huiles de palme, de palmiste, matière grasse laitière anhydre, graisse de bœuf, etc.).

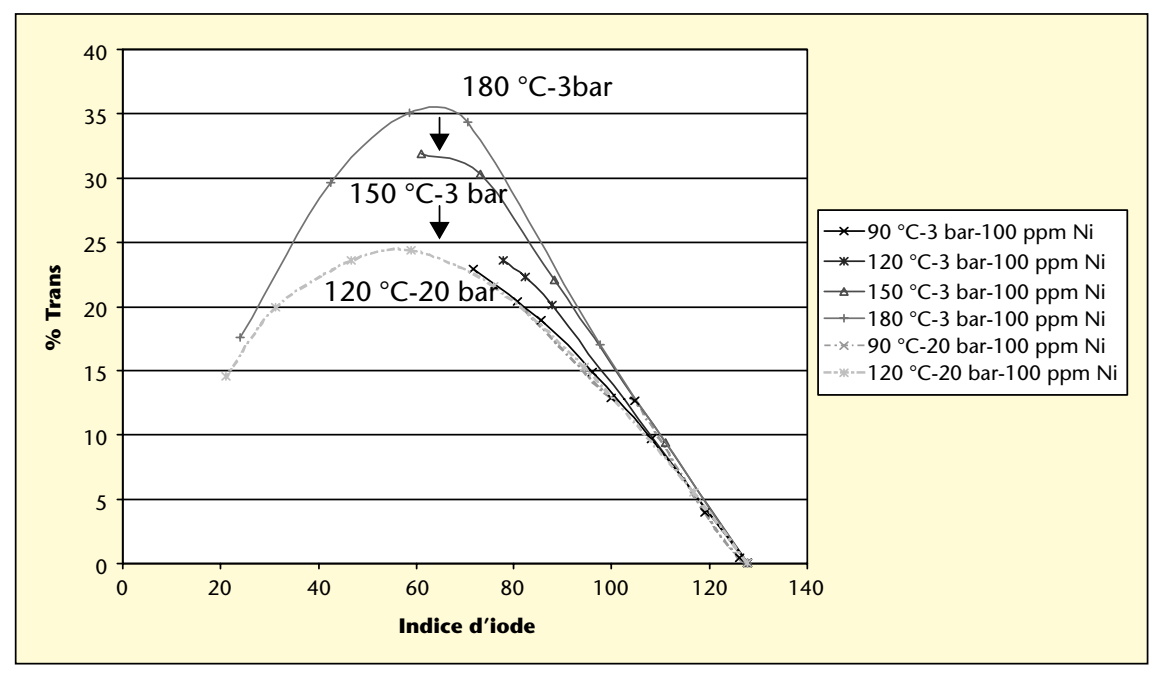

Figure 4. Influence de la température et de la pression en hydrogène sur la formation d'acides gras trans : lors d'une hydrogénation partielle d'huile de soja (catalyseur : 100 ppm de nickel) [4]. 
Tableau 3. Influence de la température et de la pression en hydrogène sur le niveau d'acides gras trans d'une huile de soja partiellement hydrogénée [4].

\begin{tabular}{|lcccc|}
\hline & A & B & C & D \\
\hline Température $\left({ }^{\circ} \mathrm{C}\right)$ & 80 & 80 & 180 & 180 \\
Concentration en catalyseur (ppm Ni) & 250 & 262 & 247 & 247 \\
Pression en hydrogène (bar) & 3,75 & 23 & 2,5 & 23 \\
II (indice d'iode) & 70,6 & 65,1 & 71,1 & 60,9 \\
Acides gras libres (\%) & 0,63 & 0,36 & 0,36 & 0,31 \\
AGT (\%) & $\mathbf{1 8 , 9}$ & $\mathbf{1 1 , 9}$ & $\mathbf{3 5 , 4}$ & $\mathbf{4 3 , 0}$ \\
C18 :1, trans (\%) & 13,8 & 10,4 & 33,1 & 42,8 \\
Augmentation en C18 :0 (\%) & 13,2 & 19,2 & 2,4 & 13,4 \\
Temps de réaction (min.) & 499 & 223 & 25 & 2,5 \\
\hline
\end{tabular}

À côté de l'interestérification chimique, l'interestérification enzymatique s'avère être une option très attractive. L'interestérification enzymatique est un procédé continu et relativement simple dans son principe qui nécessite le passage de la matière grasse sur un enzyme spécifique généralement immobilisé sur un support fixe. L'enzyme Lypozyme TL IM, une lipase issue de Theromyces Lanuginosus, constitue une option de travail attractive [5]. Immobilisé, l'enzyme est plus stable et présente une durée de vie plus longue ; il offre dès lors l'avantage de pouvoir être réutilisé, ce qui est économiquement avantageux. On ne le retrouve pas dans le produit fini, même à l'état de traces.
L'hydrogénation totale reste bien sûr l'option de choix pour la formulation de produits zérotrans.

\section{Interestérification}

La redistribution des acides gras sur le noyau glycérol par action catalytique est le principe de base de l'interestérification. L'interestérification modifie le profil et/ou le point de fusion, améliore la compatibilité entre les différents triglycérides ainsi que la plasticité de la matière. Le procédé peut être mené en catalyse chimique (interestérification aléatoire et interestérification dirigée) ou en catalyse enzymatique. L'interestérification chimique aléatoire a lieu à une température fixe $\left(70-140{ }^{\circ} \mathrm{C}\right)$, avec un temps de réaction court (classiquement 30 minutes) et une consommation faible en catalyseur (couramment 0,05-0,1\% de méthoxyde de sodium). C'est un procédé non sélectif qui agit suivant les lois de la probabilité. L'interestérification chimique dirigée est soumise à un contrôle de température en cours de procédé dans une échelle beaucoup plus basse (20-60 $\left.{ }^{\circ} \mathrm{C}\right)$. En conséquence, le temps de réaction est plus long et la consommation en catalyseur accrue. II s'agit dans ce cas d'une ségrégation sélectivement orientée par les propriétés de cristallisation de la matière.

Le désavantage de l'interestérification chimique concerne surtout les pertes en huile relativement élevées; outre les pertes inhérentes au procédé (production d'esters méthyliques et d'acide gras libres), des pertes supplémentaires liées aux post-décoloration et postdésodorisation sont à prendre en compte (les pertes totales en huile sont de $1,5 \%$ minimum sur base de $0,1 \%$ de catalyseur et $0,5 \%$ de terres de décoloration).

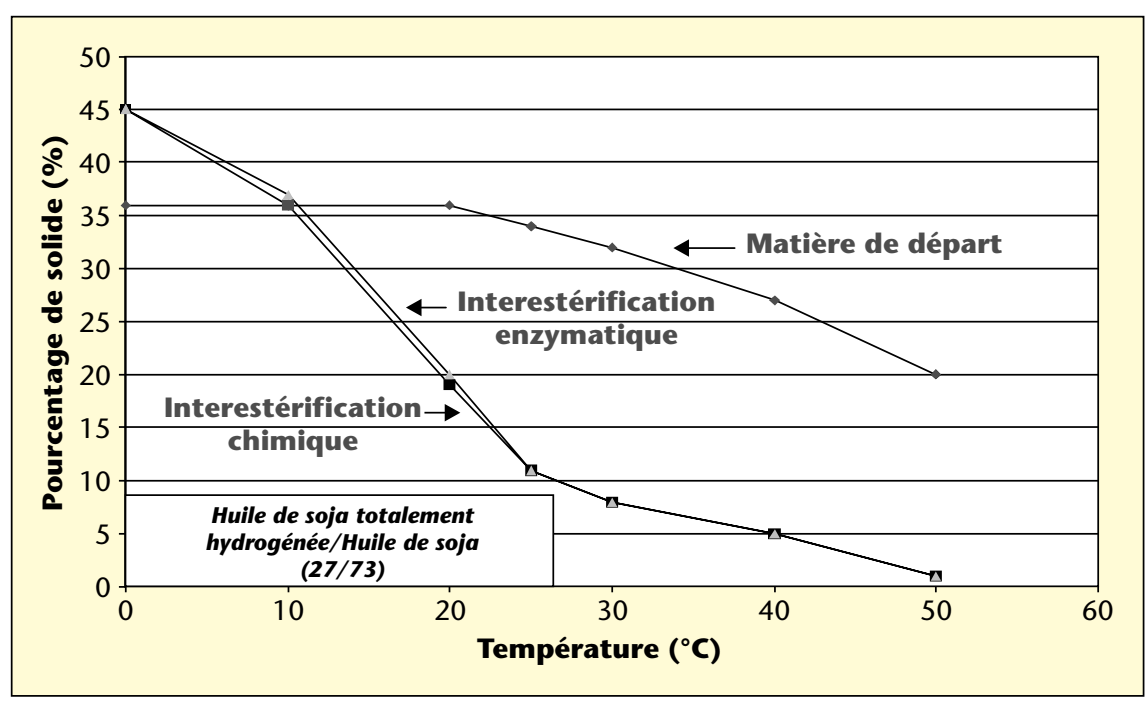

Figure 5. Pourcentage de solide (\%) de mélanges interestérifiés chimiquement et enzymatiquement (huile de soja totalement hydrogénée et huile de soja) [2].

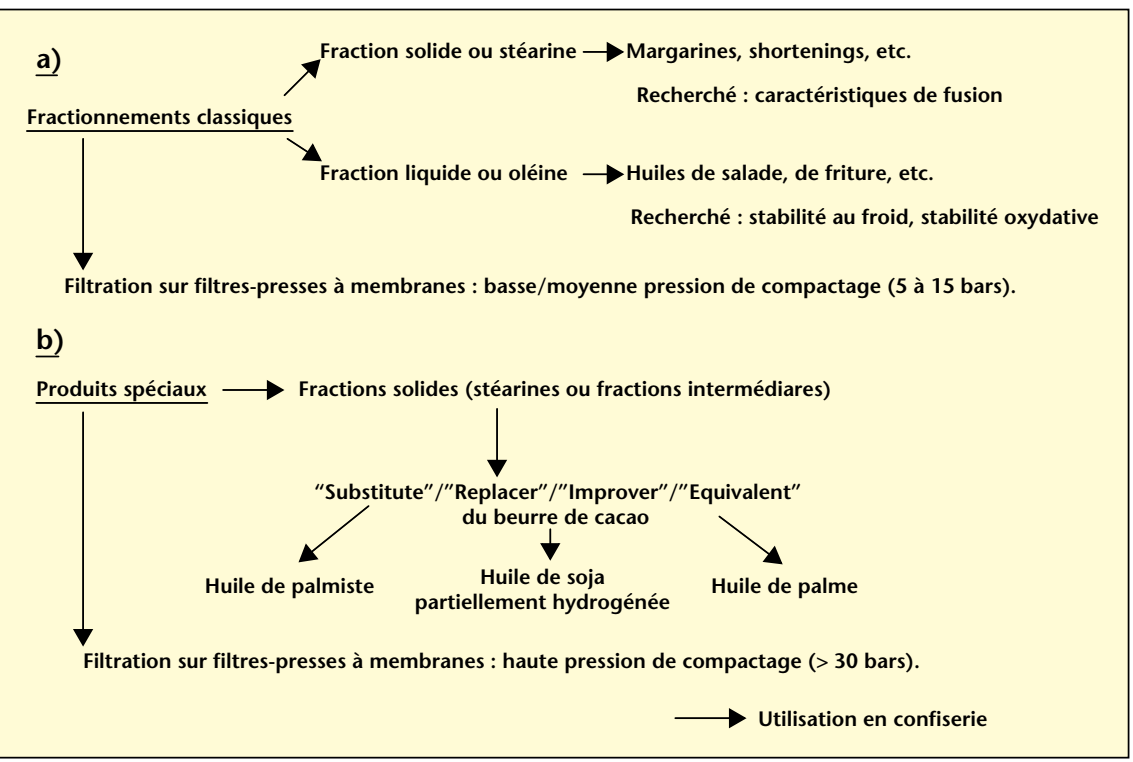

Figure 6. Démarches du fractionnement à sec : a) le fractionnement simple pour l'obtention d'une stéarine et d'une oléine et $b$ ) le façonnage de produits spéciaux par fractionnements multiples. 
Tableau 4. Avantages et désavantages liés aux procédés d'interestérification chimique et enzymatique.

\begin{tabular}{|c|c|c|}
\hline & Avantages & Désavantages \\
\hline $\begin{array}{l}\text { Interestérification } \\
\text { chimique }\end{array}$ & $\begin{array}{l}\text { Totalement aléatoire, le produit final } \\
\text { étant déterminé par la composition en } \\
\text { acides gras du mélange. } \\
\text { Procédé relativement simple et } \\
\text { reproductible réalisé en batch }\end{array}$ & $\begin{array}{l}\text { Risques au niveau manipulation du } \\
\text { catalyseur. } \\
\text { Post-traitement nécessaire dû à des } \\
\text { réactions secondaires. } \\
\text { Risque de réversion de goût et de } \\
\text { réduction de stabilité oxydative. } \\
\text { Perte en composés mineurs } \\
\text { (antioxydants). }\end{array}$ \\
\hline $\begin{array}{l}\text { Interestérification } \\
\text { enzymatique }\end{array}$ & $\begin{array}{l}\text { Simple, propre et sans risque. } \\
\text { Pas de réactions secondaires et donc } \\
\text { pas de post-traitement. } \\
\text { Pas de contact avec des agents } \\
\text { chimiques. } \\
\text { Coûts d'investissement moindres. } \\
\text { Flexible car possibilité d'opération en } \\
\text { batch ou en continu. } \\
\text { Peu de pertes en composés mineurs } \\
\text { (antioxydants). }\end{array}$ & $\begin{array}{l}\text { Catalyseur cher et sensible } \\
\text { (température, humidité, éléments } \\
\text { peroxydés, phospholipides, } \\
\text { impuretés). } \\
\text { Pré-traitement de l'huile } \\
\text { indispensable. } \\
\text { Moins flexible au niveau des } \\
\text { changements de produits } \\
\text { (contamination croisée). }\end{array}$ \\
\hline
\end{tabular}

L'enzyme Lypozyme TL IM est cacatérisé par une température optimale de travail de l'ordre de $70{ }^{\circ} \mathrm{C}$; il est sensible aux hydroperoxydes, aux impuretés polaires (phospholipides et savons), aux produits d'oxydation secondaires (aldéhydes et cétones) et aux éléments en traces tels que le nickel. L'huile à traiter sera donc préalablement dégommée, blanchie et désodorisée. La productivité enzymatique représente la quantité d'huile interestérifiée produite par $\mathrm{kg}$ d'enzyme ( $\mathrm{kg}$ huile/ $\mathrm{kg}$ enzyme). Les garanties minimales pour une huile de bonne qualité sont de l'ordre de $2500 \mathrm{~kg}$ huile/ $\mathrm{kg}$ enzyme; en pilote, il est possible $d^{\prime}$ atteindre des productivités de l'ordre de $4000 \mathrm{~kg}$ huile/kg enzyme. L'interestérification enzymatique est une réaction relativement lente en termes de débit en huile (1-2 kg huile/ $\mathrm{kg}$ enzyme* heure); par contre, elle peut être fonctionnelle pendant plus de 100 jours et ce sans interruption. Plusieurs réacteurs en série permettent d'améliorer les conditions opérationnelles.

Contrairement à l'interestérification chimique qui s'opère classiquement en batch, l'interestérification enzymatique peut se faire soit en batch soit en opération continue, en présence $d^{\prime}$ un ou de plusieurs réacteurs, ce qui lui confère un caractère plus flexible.

Un degré de conversion maximal comparable à celui de l'interestérification chimique peut être atteint par interestérification enzymatique ; les profils de fusions en fin de réaction sont en effet très proches [2] (figure 5). Parmi les effets positifs de la qualité de l'huile interestérifiée, nous retiendrons un meilleur maintien du taux de tocophérols, moins de diglycérides formés et peu de variation de couleur. En réalité, les interestérifications chimique et enzymatique présentent l'une et l'autre une série d'avanta- ges et de désavantages qui sont résumés au tableau 4. Les coûts totaux par tonne d'huile traitée sont assez similaires, les coûts opérationnels de l'interestérification enzymatique compensant totalement les coûts d'investissement et les pertes en huile importantes de l'interestérification chimique. Comparés à l'hydrogénation, les procédés d'interestérification demeurant cependant moins coûteux.

\section{Fractionnement à sec}

Le fractionnement à sec consiste en une cristallisation sélective des composants triglycéridiques à hauts points de fusion dans des maturateurs soumis à un refroidissement contrôlé, suivie d'une séparation par filtration (préféren- tiellement sur filtres-presses à membrane) et ce, en l'absence de tout adjuvant chimique ou de solvant. Cette particularité fait du fractionnement à sec une technologie verte et particulièrement économique.

Si la matière grasse de départ ne contient pas $d$ 'isomères trans, les produits fractionnés en sont également exempts.

Le fractionnement à sec est applicable à une large gamme de matières grasses: huile de palme, huile de palmiste, matière grasse laitière anhydre, huile de poisson, suif, etc. Dans tous les cas, le but recherché n'est pas simplement de produire une stéarine et une oléine, mais l'attention des industriels se focalise plutôt sur l'obtention de produits plus spéciaux (figure 6). Le fractionnement multiple de I'huile de palme est probablement le plus impressionnant du fait de la multiplicité des fractions qu'il est possible de générer (figure 7). La route liquide permet de produire en plusieurs étapes des oléine, superoléine et topoléine d'indices d'iode croissants et de tenue au froid améliorée tandis que la route solide produit des stéarines aux propriétés multiples. La Soft Palm Mid Fraction (Soft PMF) par exemple est un ingrédient de choix pour la production de margarines et de shortenings. Le refractionnement de cette Soft PMF produit une Hard PMF, excellent ingrédient d'équivalent de beurre de cacao ; sa richesse en acides palmitique, stéarique et oléique lui confère en effet une bonne compatibilité avec le beurre de cacao en plus d'un profil de fusion particulièrement raide entre 20 et $30{ }^{\circ} \mathrm{C}$.

Le fractionnement à sec permet donc de générer une large gamme de produits aux applications diverses: huiles de friture et de cuisson, huiles de salade, margarines, shortenings, pro-

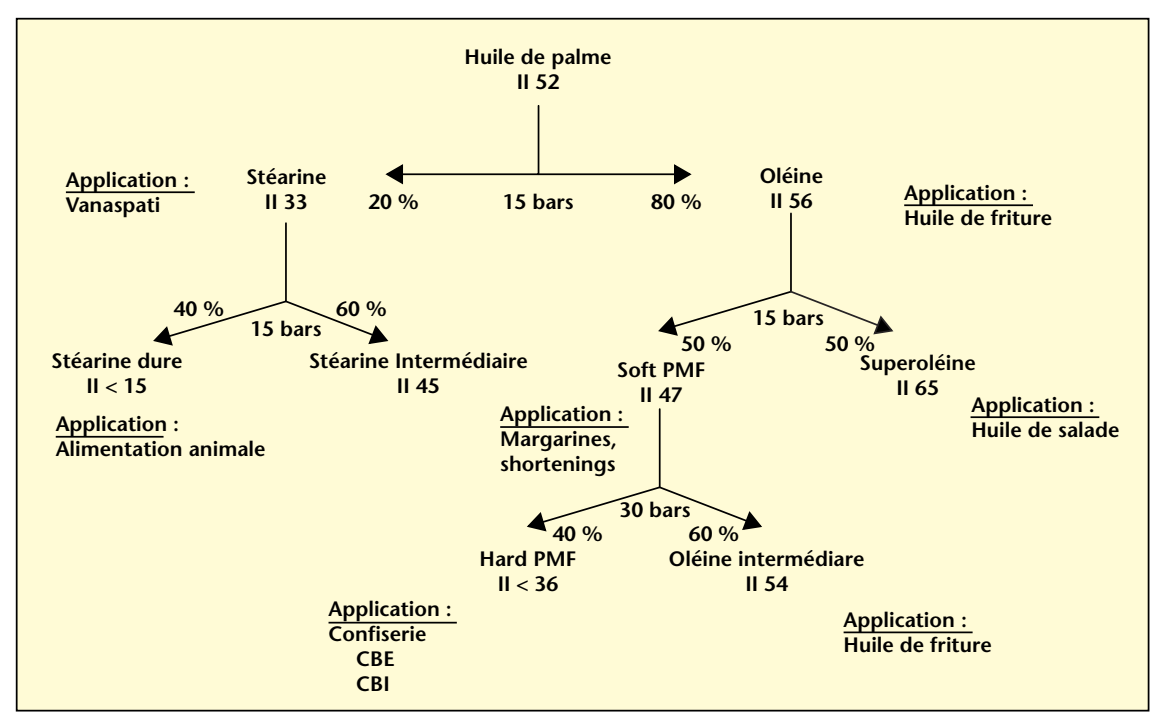

Figure 7. Fractionnement multiple de I'huile de palme (II : Indice d'iode).

$C B E$ : « Equivalent » du beurre de cacao. $C B I$ : «Improver » du beurre de cacao. 


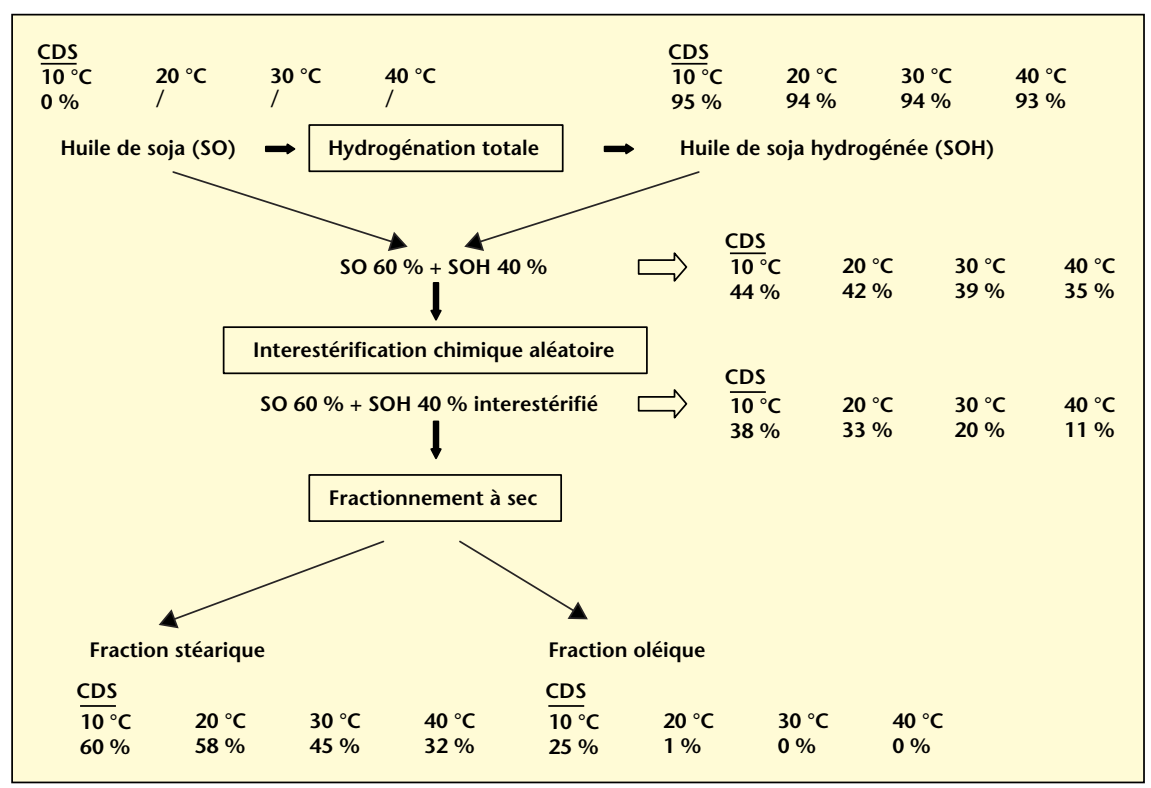

Figure 8. Exemple de combinaisons des trois technologies de modification : hydrogénation totale, interestérification chimique aléatoire et fractionnement à sec pour la formulation de nouveaux produits de type zéro-trans [6].

CDS : courbe de solidification (pourcentage de solide)

duits de confiserie, graisses de fourrage, d'enrobage, etc. II s'agit bien sûr d'une technologie multifonctionnelle qui constitue définitivement le procédé de modification du futur. Son utilisation en combinaison avec des techniques d'hydrogénation et d'interestérification le positionne en tête des technologies de transformation.

De vastes gammes d'alternatives à bas taux $d$ 'isomères trans peuvent ainsi être produites par combinaison de chacuns des trois procédés.

Par exemple, des fractions dures issues de I'hydrogénation totale (huile de soja totale-
Le fractionnement à sec et l'interestérification chimique et enzymatique constituent des techniques de choix permettant à terme de remplacer l'hydrogénation partielle. La formulation de produits au label zéro-trans est désormais possible par combinaison de ces techniques de modification (y inclus l'hydrogénation totale).

\section{RÉFÉRENCES}

1. MAES I, DE MEULENAER B, VAN HEERSWYNGHELS P, DE GREYT W, EPPE G, DE PAUW E, HUYGHEBAERT A. Removal of dioxins and PCB from fish oil by activated carbon and its influence on the nutritional quality. I Am Oil Chem Soc $2005 ; 82(8)$ : 593-7.

2. DESMET BALLESTRA. Données internes.

3. KELLENS M. Etat des lieux et évaluation des procédés de modification des matières grasses par combinaison de l'hydrogénation, de l'interestérification et du fractionnement. $O C L$ $1998 ; 5(5)$ : 384-91.

ment hydrogénée) ou du fractionnement à sec (stéarine de palme) peuvent être mélangées à des huiles fluides (huile de soja en l'état), en proportions variables et ensuite interestérifiées. Un fractionnement à sec peut venir parfaire les caractéristiques du produit final [6] (figure 8).

\section{Conclusion}

Les agents contaminants peuvent être efficacement extraits lors des étapes de raffinage avec une attention toute spéciale envers le traitement au charbon actif et les conditions de désodorisation.
4. DELAHAUT S. Optimisation des conditions opératoires de l'hydrogénation sélective de I'huile de soja. Travail de fin d'études. Faculté des Sciences Appliquées, Université Catholique de Louvain, 1999.

5. NOVOZYMES A/S, Vadstrupvej $41-43$, bld. 7a 2880 Bagsværd Denmark.

6. KELLENS M. Etat des lieux et évaluation des procédés de modification des matières grasses par combinaison de l'hydrogénation, de l'interestérification et du fractionnement (suite). OCL 1998 ; 5(6) : 421-6. 